\title{
Une collection de musique populaire suisse
}

\section{Michele L. Straniero}

Traducteur : Georges Goormaghtigh

\section{(2) OpenEdition}

\section{Journals}

Édition électronique

URL : http://journals.openedition.org/ethnomusicologie/2413

ISSN : 2235-7688

\section{Éditeur}

ADEM - Ateliers d'ethnomusicologie

\section{Édition imprimée}

Date de publication : 1 janvier 1990

Pagination : 254-259

ISBN : 2-8257-0423-7

ISSN : 1662-372X

\section{Référence électronique}

Michele L. Straniero, "Une collection de musique populaire suisse », Cahiers d'ethnomusicologie [En ligne], 3 | 1990, mis en ligne le 15 octobre 2011, consulté le 05 mai 2019. URL : http://

journals.openedition.org/ethnomusicologie/2413

Ce document a été généré automatiquement le 5 mai 2019.

Article L.111-1 du Code de la propriété intellectuelle. 


\title{
Une collection de musique populaire suisse
}

\author{
Michele L. Straniero \\ Traduction : Georges Goormaghtigh
}

\section{RÉFÉRENCE}

Canti liturgici popolari nel Ticino. Liturgia eucaristica e dell'ufficiatura funebre (enregistrements Arnold Geering, 1949-1952); Vespro 1983, enregistrements Pietro Bianchi, Texte de présentation: P. Bianchi. Un microsillon Fata Morgana FM 84022, 1984. Der Volksliedsänger und -forscher Hanns in der Gand. Enregistrements 1925-1934. Texte de présentation : Christine Burckhardt-Seebass. Un microsillon Fata Morgana FM 84023, 1984.

Musique populaire suisse. Collection Constantin Brăiloiu. Chansons profanes, chants religieux, danses et airs instrumentaux, musique des coutumes du cycle annuel, chansons et formulettes enfantines. Enregistrements 1927-1951. Textes de présentation : Laurent Aubert, Brigitte Bachmann-Geiser, Conrad Beck, Pietro Bianchi, Christine BurckhardtSeebass. Deux microsillons VDE-Gallo VDE 30-477/78, 1986. [En collaboration avec les Archives internationales de musique populaire du Musée d'ethnographie de Genève]. Chante Jura. Enregistrements 1952-1985. Textes de présentation : Gaston Bra-hier, Christine Burckhardt-Seebass, Barbara Eng. Un microsillon Fata Morgana FM 85736, 1988.

Canti liturgici popolari nel Ticino. Liturgia eucaristica e dell'ufficiatura funebre (enregistrements Arnold Geering, 1949-1952) ; Vespro 1983, enregistrements Pietro Bianchi, Texte de présentation: P. Bianchi. Un microsillon Fata Morgana FM 84022, 1984. Der Volksliedsänger und -forscher Hanns in der Gand. Enregistrements 1925-1934. Texte de présentation : Christine Burckhardt-Seebass. Un microsillon Fata Morgana FM 84023, 1984.

Musique populaire suisse. Collection Constantin Brăiloiu. Chansons profanes, chants religieux, danses et airs instrumentaux, musique des coutumes du cycle annuel, chansons 
et formulettes enfantines. Enregistrements 1927-1951. Textes de présentation : Laurent Aubert, Brigitte Bachmann-Geiser, Conrad Beck, Pietro Bianchi, Christine BurckhardtSeebass. Deux microsillons VDE-Gallo VDE 30-477/78, 1986. [En collaboration avec les Archives internationales de musique populaire du Musée d'ethnographie de Genève]. Chante Jura. Enregistrements 1952-1985. Textes de présentation : Gaston Bra-hier, Christine Burckhardt-Seebass, Barbara Eng. Un microsillon Fata Morgana FM 85736, 1988.

1 Ces cinq microsillons constituent un excellent début à la nouvelle collection discographique publiée dès 1984 par la Société suisse des traditions populaires (le troisième disque comporte deux disques dans un coffret). L'édition est préparée de manière exemplaire du point de vue critique; chaque album comprend un fascicule illustrant abondamment le contenu par des textes et des notes. Le choix du répertoire tend d'ores et déjà à se distinguer par l'ambition de couvrir les divers aspects de la recherche folklorique dans le domaine musical, pour tout le territoire suisse.

2 Canti liturgici popolari nel Ticino, signé par Pietro Bianchi, met au grand jour une série d'enregistrements monophoniques du répertoire liturgique et para-liturgique populaire effectués entre 1949 et 1952 par Arnold Geering, alors directeur des Archives suisses des traditions populaires à Bâle. Cette série est complétée par des enregistrements sur le même thème réalisés par Bianchi en 1983. La campagne de recherche de Geering fut suivie pas à pas par don Luigi Agustoni, un expert en chant grégorien, qui ne manqua pas de souligner - dans un article paru en 1950 dans Folklore suisse - l'étroite parenté de certains de ces chants avec l'ancienne psalmodie de l'Église romaine et ambrosienne, appelée conventionnellement " grégorienne », quoi que cela puisse signifier.

3 Geering et Agustoni, nous explique Bianchi, enregistrèrent les chants qui accompagnent la célébration de la messe en omettant ceux de l'office des morts, les processions et les Vêpres; ils y inclurent cependant les chants du très riche répertoire mariai. Malheureusement, dans bien des cas - déplore très justement l'éditeur - les enregistrements de Geering ne comportent que les premières phrases d'un chant : ainsi son choix a-t-il été guidé par un sens esthétique et philologique très sûr, retenant les Gloria, Credo, Sanctus et Agnus d'Arosio, pour leur caractère exhaustif, en les complétant par le Kyrie d'Isone, exécuté par les seules voix masculines, puisqu'en 1820, les femmes de cette localité ne chantaient pas à l'église. Viennent ensuite les différentes versions du psaume cinquante (Miserere) et de la Lamentation de Jérémie, un Dies Irae de Cavergno et, sur la seconde face, les chants du Vespéral enregistrés par Bianchi en 1983, avec le très beau Magnificat de Sementina et le Mysterium Ecclesiae enregistrés à Ponto Valentino, précédé par les tambours de la Milice, pour répondre à un vœu fait par les mercenaires tessinois au cours de l'expédition de Napoléon en Russie.

4 La documentation sur ces survivances du chant liturgique populaire en latin est précieuse après le coup d'éponge radical du Concile Vatican II. Elle devrait être étendue à d'autres zones, comme du reste cela se fait déjà en Italie, surtout à l'initiative de quelques rares amateurs isolés et méritants qui ont obtenu des résultats remarquables : je pense, par exemple, aux extraordinaires enregistrements effectués et publiés par Mario Rigoldi à Carugate, en Brianza, que j'ai présenté moi-même avec intérêt et une grande émotion aussi bien à la Radio suisse qu'à Radio Vatican, dans un document intitulé justement Les cloches de Carugate (1985). La vigueur de ces voix et de ces mélodies populaires est irremplaçable, et leurs qualités esthétiques et expressives sont sans comparaison avec tout ce qui a été fait au cours des vingt dernières années par des compositeurs, même très 
doués et pleins de bonne volonté, pour la «nouvelle musique » liturgique de l'Église romaine ${ }^{1}$.

5 Le chanteur Hanns in der Gand (1882-1947), pseudonyme ou nom d'artiste de Ladislaus Krupski, fut un protagoniste important de la chanson suisse de l'entre-deux-guerres et un pionnier de l'ethnomusicologie helvétique. Le disque qui lui est consacré, deuxième de la série dont il est question ici, a été édité par les soins de Christine Burckhardt-Seebass; il met en relief sa personnalité forte et complexe de musicien, de chanteur et de chercheur. Une notice biographique publiée dans l'intéressant fascicule d'accompagnement nous renseigne sur les origines polonaises de Krupski, né à Ernstfeld, dans le canton d'Uri, d'un père médecin échappé des prisons russes et d'une mère née, elle, dans le canton de Berne.

cours de ses études au Gymnase de Lucerne, il eut l'occasion d'entrer en contact avec deux éminentes personnalités de la culture suisse, Renward Branstetter et Theodor von Liebenau, qui lui donnèrent le goût des collections et des archives, et firent de lui un chercheur passionné. Ce choix fut certainement aussi dicté par le fait que la mère de Hanns - une Huggler de Brienzwiler - était une excellente chanteuse populaire, comme le sont, par tradition familiale, de nombreuses femmes de ce village.

7 La période de formation de Hanns in der Gand (l'origine de ce nom d'artiste ne nous est pas expliquée) coïncide avec la fameuse redécouverte de la chanson populaire suisse, œuvre d'Alfred Leonz Gassmann et Otto von Greyenz, alors qu'en Allemagne les mouvements connus sous le nom de "Wandervogel » et "Jugendbewegung" travaillaient dans la même perspective culturelle. En 1914, à la veille de la première guerre mondiale, le jeune ténor et luthiste (il avait fait ses études musicales en Allemagne) était déjà un célèbre interprète des ballades populaires traditionnelles qu'il avait fait connaître aussi bien dans sa patrie qu'à l'étranger. A ce titre, il fut remarqué par le commandant en chef de l'armée suisse, le général Wille, qui voulut l'engager comme chanteur officiel de ladite armée!

8 En 1917, Hanns publia un recueil de Vieilles chansons populaires et militaires de la suisse romande et italienne - auquel s'ajoutèrent avec le temps les Alti Schwyzerlieder (1921) et la Scella di canzoni popolari ticinesi en 1933. Entre-temps, il composa des chansons qui connurent un grand succès et il effectua de nombreuses tournées de chant, en Suisse comme à l'étranger. Il visita aussi plusieurs colonies suisse du Nouveau Monde, toujours à la recherche de vieilles chansons dont les groupes d'émigrants restés unis sont de précieux dépositaires. C'est pendant cette période qu'il publia des disques dont une sélection est proposée dans cette anthologie. A partir de 1930, in der Gand collabore étroitement avec la Société suisse des traditions populaires, effectuant des recherches de terrain systématiques un peu partout dans le pays.

9 Hanns in der Gand mourut en 1947, à l'âge de 65 ans. Ses recueils de chants et de musiques réalisés pour la SSTP se trouvent à Bâle, à la disposition des spécialistes, alors que ses collections personnelles demeurent la propriété de sa famille. Les quinze morceaux figurant dans le disque comportent, sur la face $\mathrm{A}$, une série de chants badins $\mathrm{du}$ répertoire de bistrot du canton d'Uri (les célèbres Lumpeliedli), sur une thématique locale, la fête de la Chil-bi, et un deuxième ensemble de chants humoristiques liés à des coutumes populaires. La face B, en revanche, présente un groupe substantiel de chants guerriers qui impressionèrent profondément la génération de l'époque de la Première Guerre mondiale, suivi d'un exemple de Heimwehlied, tiré du répertoire nostalgique des émigrants, ainsi que de deux compositions d'in der Gand lui-même, elles aussi de 
caractère militaire, qui rencontrèrent un grand succès pendant la Grande Guerre, digne couronnement d'une édition discographique vraiment exemplaire.

Fritz Walter, joueur de Hackbrett

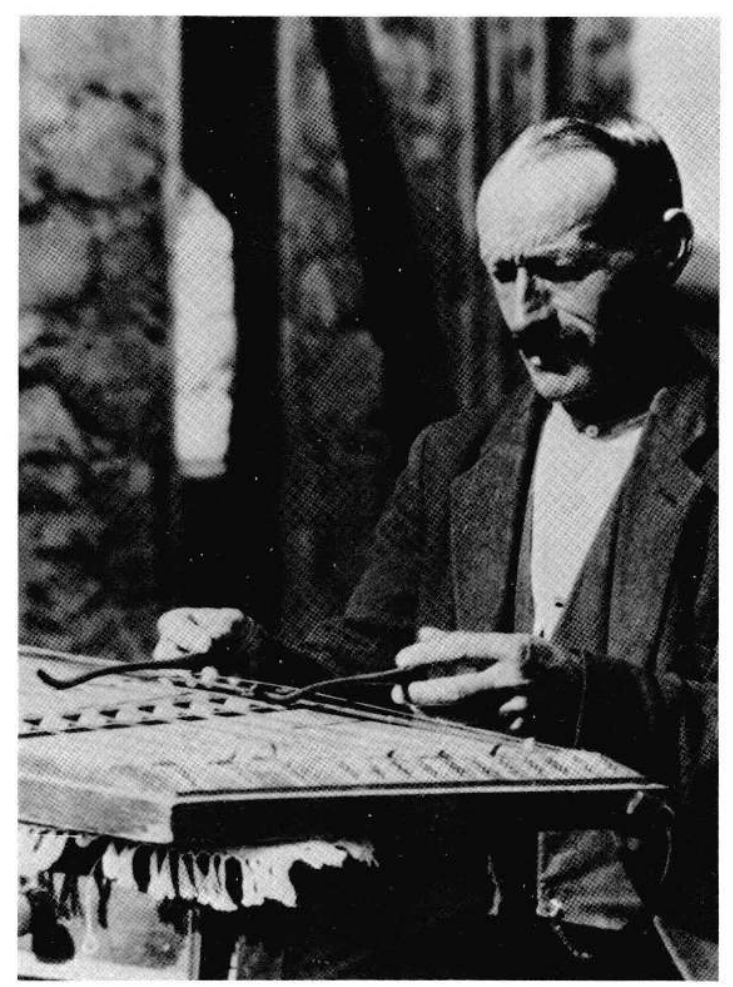

Gräschen, Valais, Suisse. Photo : Archives SSTP.

Il pourrait sembler superflu de souligner l'importance capitale du recueil que constitue Musique populaire suisse, Collection Constantin Brăiloiu, établi sur la base d'enregistrements effectués entre 1927 et 1951 par le grand folkloriste roumain. Installé à Genève à partir de 1944, il y fonda les Archives internationales de musique populaire (AIMP) dans le cadre du Musée d'ethnographie. Cette réédition sur microsillon a été menée conjointement par Laurent Aubert et Christine Burckhardt-Seebass, avec la collaboration scientifique de Brigitte Bachmann-Geiser et de Pietro Bianchi ; elle offre à l'auditeur passionné comme au spécialiste un riche aperçu de soixante-cinq pièces couvrant une aire qui s'étend du Tesssin à Appenzell, de l'Argovie à Saint Gall sans négliger le canton historique de Schwyz. Elle fournit ainsi un panorama articulé et savoureux de cet abondant répertoire. Laurent Aubert en souligne justement la très soigneuse sélection qui tend à mettre en relief ce que Brăiloiu appelait "les processus historiques de développement », faisant allusion aux degrés et aux modes successifs de pénétration d'éléments modernes dans le tissu musical rural traditionnel. Là encore, un excellent travail critique a été fait dans le fascicule joint au recueil. Celui-ci comporte de nombreuses notices introductives sur les différents aspects et sur la thématique du travail de Brăiloiu, ainsi que des textes et des notes sur toutes les pièces reproduites, qui en font d'emblée un classique de la discographie ethnomusicologique.

11 Chante Jura, le cinquième microsillon de la série, est en grande partie le fruit des recherches de terrain menées par Barbara Eng au cours des années 1983-1984. Il comporte, en outre, quelques enregistrements moins récents (les plus anciens remontant 
à 1952). Ce beau disque est présenté officiellement par Gaston Brahier, ministre de l'Éducation et des Affaires sociales de la République et Canton du Jura, qui se félicite de la promptitude et de la sensibilité avec lesquelles la constitution cantonale a répondu à l'intérêt public pour la conservation et la valorisation du patrimoine traditionnel local. Une note introductive riche et documentée de l'éditrice Barbara Eng décrit les vicissitudes de la recherche au Jura, amorcée par le linguiste Arthur Rossat en 1894 et poursuivie dans les premières années de ce siècle par les chercheurs de la Société jurassienne d'émulation. En 1952 commencèrent les recherches menées par les ethnomusicologues Arnold Geering (appelé ici Adolf par erreur) et Zygmunt Estreicher qui enregistrèrent une cinquantaine de morceaux à Porrentruy. Quatre exemples de cette recherche sont insérés dans le disque.

En 1983, les Archives suisses de la chanson populaire chargèrent Barbara Eng, étudiante en musicologie et en ethnologie à Bâle, d'effectuer une étude sur le répertoire de la jeune République; au cours de ses recherches, elle eut la chance de rencontrer Marguerite L'Hoste, qui avait déjà chanté pour Geering et Estreicher en 1952, renouvelant ainsi l'apport de son précieux témoignage: un événement bien rare dans les annales folkloriques et d'une grande importance méthodologique pour les confirmations et les contrôles qu'il permet. Une année de recherches sur le terrain a fourni à Madame Eng un ensemble d'environ cent cinquante ballades et chansons, qui illustrent l'évolution du répertoire dans le canton, après sa séparation d'avec Berne (événement qui a provoqué un retour aux anciennes traditions en patois local). Ainsi, pour réaliser ce disque, il n'y eut que l'embarras du choix.

\section{NOTES}

1. Pour un examen de la question, voir mon ouvrage intitulé Mira il tuo pop : origine e peripezie del canto cristiano. Milan : Oscar Mondadori, 1989. 